\title{
Experimental infection of gnotobiotic pigs with the cell-culture-adapted porcine deltacoronavirus strain OH-FD22
}

\author{
Hui $\mathrm{Hu}^{1,2} \cdot$ Kwonil Jung $^{1} \cdot$ Anastasia N. Vlasova $^{1} \cdot$ Linda J. Saif $^{1}$
}

Received: 22 July 2016/Accepted: 7 September 2016/Published online: 12 September 2016

(C) Springer-Verlag Wien 2016

\begin{abstract}
Porcine deltacoronavirus (PDCoV) is a novel enteropathogenic coronavirus in pigs. We have isolated and passaged the PDCoV strain OH-FD22 in an LLC porcine kidney (LLC-PK) cell line. Our study investigated the pathogenicity of the tissue-culture-grown PDCoV (TCPDCoV) OH-FD22 at cell passages 5, 20 and 40 in LLCPK cells, in eight 14-day-old gnotobiotic (Gn) pigs. Pigs $(\mathrm{n}=3)$ were euthanized for pathologic examination at post-inoculation day (PID) 3, and the remainder were monitored for clinical signs, virus shedding, and serum antibody responses until PID 28. All inoculated pigs developed watery diarrhea and/or vomiting at PID 1-2 and shed the highest amount of viral RNA in feces at PID 3-5, accompanied by severe atrophic enteritis. They developed high titers of PDCoV-specific IgG/IgA and virus-neutralizing antibodies in serum at PID 23-24. Histologic lesions were limited to the villous epithelium of the jejunum and ileum at PID 3. Two inoculated pigs tested at PID 23-24 had small to moderate numbers of PDCoV antigen-positive cells in the intestinal lamina propria and mesenteric lymph nodes, but not in enterocytes. An analysis of full-length $\mathrm{S}$ and $\mathrm{N}$ genes of TC- and Gn-pig-passaged OH-FD22 revealed a high genetic stability in cell culture and pigs. TC-PDCoV OH-FD22 (cell passages 5, 20 and 40) was enteropathogenic, and the pathogenicity was similar to that
\end{abstract}

Linda J. Saif

saif.2@osu.edu

1 Food Animal Health Research Program, Ohio Agricultural Research and Development Center, Department of Veterinary Preventive Medicine, The Ohio State University, 1680 Madison Ave., Wooster, OH 44691, USA

2 College of Animal Science and Veterinary Medicine, Henan Agricultural University, Zhengzhou 450002, China of the original field virus. The TC-PDCoV OH-FD22 will be useful for further pathogenesis studies and for evaluating if higher-level cell-culture passaged virus becomes attenuated for vaccine development.

\section{Introduction}

Porcine deltacoronavirus (PDCoV), belonging to the genus Deltacoronavirus of the family Coronaviridae [27], is a porcine diarrheal pathogen that was initially reported in Hong Kong in 2012 [28] and emerged in the United States in 2014 [20, 25, 28]. Infected herds had clinical signs of acute watery diarrhea in sows and nursing pigs, but mortality was reported only in nursing pigs $[4,12,25]$. PDCoV disease was reportedly milder than that caused by porcine epidemic diarrhea virus (PEDV) and transmissible gastroenteritis virus (TGEV) in seronegative herds [13, 17]. Molecular surveillance studies indicated that PDCoV coinfections are common, especially with rotavirus group $\mathrm{C}$ (Rota C) and PEDV [7, 20]. To date, outbreaks have been documented in more than 20 states in the United States and in Canada, China, South Korea, and Thailand [6, 8, 15, 23]. A report from Jiangxi Province, China, indicated that the prevalence of monoinfection by $\mathrm{PDCoV}$ was high $(33.7 \%)$, and coinfection by PDCoV and PEDV was $19.7 \%$ in diarrheic pigs [23].

Several investigators have described the molecular detection and genetic analysis of PDCoVs $[15,16,19$, $22,24]$. Other studies confirmed that PDCoV is enteropathogenic in young pigs $[4,12,17]$. Our previous study showed severe watery diarrhea and/or vomiting and severe atrophic enteritis in 14-day-old gnotobiotic (Gn) pigs inoculated with the PDCoV OH-FD22 original fecal sample [12]. Another study reported that a cell culture isolate 
of PDCoV, USA/IL/2014, caused clinical disease in conventional 5-day-old piglets, accompanied by macroscopic and microscopic lesions in the small intestine [4]. It was also reported that a cell culture isolate of PDCoV, strain Michigan/8977/2014 (MI), caused severe gastrointestinal diseases in Gn and conventional 10-day-old piglets [17].

In our recent publication, we focused on the isolation of PDCoV in cell culture. The PDCoV OH-FD22 strain originated from intestinal contents from a diarrheic pig, and it was successfully isolated in two cell lines of swine origin, swine testicular (ST) and LLC porcine kidney (LLC-PK) cells. It was serially passaged in LLC-PK cells [7]. The aims of our current study were to investigate i) the pathogenicity of the tissue-culture-grown PDCoV (TC$\mathrm{PDCoV}$ ) strain OH-FD22 at passage 5 (P5), P20, and P40 on LLC-PK cells in 14-day-old Gn pigs compared with that of the wild-type parent virus [12]; ii) Fecal PDCoV shedding, viremia, and pathology tested at post-inoculation day (PID) 1 to 23-24 or PID 28; iii) possible attenuation of TCPDCoV OH-FD22 P40, the highest passage in cell culture among the passages tested; iv) serum-PDCoV-specific IgG and $\mathrm{IgA}$ and virus neutralization ( $\mathrm{VN}$ ) antibody titers and any differences in the titers among OH-FD22 P5, P20, and P40-inoculated pigs; and v) the genetic stability of TC- and Gn-pig-passaged PDCoV OH-FD22 P5, P20, and P40 by analysis of the complete spike (S) and nucleocapsid $(\mathrm{N})$ gene sequences.

\section{Materials and methods}

\section{Cells and virus}

The LLC-PK cell line (ATCC CL-101) was used to serially passage the PDCoV OH-FD22 strain, which was isolated from the intestinal contents collected from a diarrheic pig from Ohio [7]. The TC-PDCoV OH-FD22 P5, P20, and P40 strains were propagated on LLC-PK cells and harvested when the CPE was $>85 \%$. Virus titer was determined by using TaqMan real-time quantitative RT-PCR (qRT-PCR) and by plaque assay as described previously [7]. The propagated PDCoV culture was confirmed negative for other swine enteric viruses, including TGEV, PEDV, Rota A to Rota C, and caliciviruses (noroviruses, sapoviruses, and St-Valerien-like viruses), by using RTPCR as reported previously [1, 2, 14, 21, 26].

\section{Inoculation of Gn pigs with TC-PDCoV OH-FD22}

The TC-PDCoV strains OH-FD22 P5, P20, and P40 in LLC-PK cells were used for inoculation of Gn pigs. All animal studies were performed as approved by the Institutional Animal Care and Use Committee (IACUC) of the
Ohio State University. The Gn pigs were delivered aseptically by hysterectomy from specific-pathogen-free sows. Prior to inoculation, pigs were confirmed negative for the major swine enteric viruses by testing of fecal samples using RT-PCR. Six 14-day-old Gn pigs $(\mathrm{n}=2$ for each passage) were inoculated orally with TC-PDCoV $\mathrm{OH}-$ FD22 P5 (10.8 $\log _{10}$ genomic equivalents [GE] per pig), P20 (10.2 $\log _{10}$ GE per pig), and P40 (10.1 $\log _{10}$ GE per pig). Two pigs of the same age were used as negative controls. Clinical signs were monitored at least twice daily [12]. For each virus passage group, one of the two pigs was euthanized for pathologic examination at 24-48 h after onset of clinical signs [12]. Large-intestinal contents (LIC) and small-intestinal contents (SIC) were collected and tested by qRT-PCR for PDCoV and by RT-PCR for other enteric viruses. The remaining pigs were monitored for longer-term clinical signs, fecal virus RNA shedding, and virus-specific serum antibodies until PID 28. Diarrhea was assessed by scoring fecal consistency as follows: $0=$ solid; $1=$ pasty; $2=$ semi-liquid; $3=$ liquid, with scores of 2 or more considered diarrheic [10].

\section{Viral RNA detection by qRT-PCR}

Rectal swabs were collected daily from each pig throughout the experiment. Viral RNA was extracted from the intestinal content suspensions, rectal swab fluids, serum, and cell culture samples by using a 5 MagMAX-96 virus isolation kit (Ambion by Life Technologies, USA) and the RNA extraction robot MagMax Express (Applied Biosystems, Foster City, CA) according to the manufacturer's instructions. Viral RNA titers were determined by qRTPCR (QIAGEN, Valencia, CA, USA) as reported [7]. The detection limit of the qRT-PCR was 10 GEs/reaction, which corresponded to 4.6 and $3.6 \log _{10} \mathrm{GE} / \mathrm{mL}$ of PDCoV in fecal and serum samples, respectively.

\section{Histopathology and immunofluorescence staining for the detection of PDCoV antigen}

Small (duodenum to ileum) and large (cecum and colon) intestinal tissues and other major organs (lung, liver, heart, kidney, spleen, and lymph nodes) were examined grossly and histologically and fixed in $10 \%$ neutral formalin for 1-2 days at room temperature [9]. They were embedded, sectioned, and stained with Mayer's hematoxylin and eosin (H\&E) for light microscopy examination. Mean jejunal ratios of villous height and crypt depth (VH:CD) were measured by using PAX-it software (PAXcam, Villa Park, IL, USA) as described previously [11]. The prepared tissues were tested by immunofluorescence (IF) staining for the detection of PDCoV antigen using a swine hyperimmune antiserum (OH-DC97) against PDCoV [7, 12]. 
Tissues from age-matched control pigs were tested for histological comparisons and as a negative control for IF.

\section{PDCoV $80 \%$ plaque reduction neutralization test (PRNT ${ }_{\text {80 }}$ )}

Neutralizing antibodies in the sera of PDCoV-inoculated pigs were tested by $\mathrm{PRNT}_{80}$ using ST cells in 6-well plates. The swine hyperimmune antiserum OH-DC97 and the mock-infected pig's serum were used as positive and negative serum controls, respectively. All sera were inactivated at $56{ }^{\circ} \mathrm{C}$ for $30 \mathrm{~min}$ prior to testing and prepared as 4-fold serial dilutions in MEM. Each sample was mixed with an equal volume of TC-PDCoV OH-FD22 (60 plaqueforming units [PFU]/300 $\mu 1$ in each well) and then incubated at $37{ }^{\circ} \mathrm{C}$ for $90 \mathrm{~min}$. The growth medium for ST cells (100\% confluent) was replaced with maintenance medium (without trypsin) as described [7]. Following $1 \mathrm{~h}$ of incubation at $37{ }^{\circ} \mathrm{C}$, cells were washed once with maintenance medium. The virus-serum mixtures were added to the ST cells, and the virus control wells were inoculated with 300 $\mu \mathrm{l}$ of PDCoV OH-FD22 (60 PFU/well). The negative control wells received the maintenance medium only. After adsorption for $1 \mathrm{~h}$ at $37{ }^{\circ} \mathrm{C}$ in an atmosphere of $5 \% \mathrm{CO}_{2}$, the inoculum was removed, and cells were washed twice with Dulbecco's phosphate-buffered saline (DPBS) without $\mathrm{Mg}^{2+}$ and $\mathrm{Ca}^{2+}$ (Sigma, St. Louis, MO). Two $\mathrm{ml}$ of overlay medium $(2 \times$ MEM [Gibco, USA] containing $1 \%$ antibiotic-antimycotic, HEPES, NEAA, and $2 \%$ pancreatin [Sigma, USA] and an equal volume of $3 \%$ SeaPlaque agarose [Lonza, Rockland, ME]) was added to each well. The plates were incubated for 2 days in an incubator $\left(37{ }^{\circ} \mathrm{C}, 5 \% \mathrm{CO}_{2}\right)$, and the plates were then stained with $0.01 \%$ neutral red (Sigma) for $2 \mathrm{~h}$ at $37^{\circ} \mathrm{C}$. The plaques were counted, and the $\mathrm{PRNT}_{80}$ was determined as the reciprocal of the highest serum dilution that neutralized $80 \%$ of the plaques as compared to that in the virus-only control wells.

\section{Serum PDCoV-specific antibody detection}

An indirect enzyme-linked immunosorbent assay (ELISA) was used to detect PDCoV-specific IgA and IgG antibodies in the sera of PDCoV-inoculated pigs. The protein concentration in the TC-PDCoV OH-FD22 was determined by a Bradford protein assay using bovine serum albumin (BSA) as a standard [3]. The 96-well MaxiSorp plates (Nunc, San Diego, CA) were coated with the TC-PDCoV OH-FD22 (100 ng/well) in coating buffer $(20 \mathrm{mM} \mathrm{Na} 2$ $\left.\mathrm{CO}_{3}, 20 \mathrm{mM} \mathrm{NaHCO} 3, \mathrm{pH} 9.6\right)$ at $4{ }^{\circ} \mathrm{C}$ overnight. The mock-infected LLC-PK cells were processed in parallel as above, and a similar concentration of antigen was used to coat the negative control wells. The wells were washed with phosphate-buffered saline (PBS) containing $0.05 \%$ Tween 20 (PBST) and then blocked with $4 \%$ non-fat dry milk in PBS. One hundred $\mu$ lof serially diluted serum (4fold dilutions) was added to the antigen-coated or mock antigen-coated wells and incubated for $1 \mathrm{~h}$ at $37{ }^{\circ} \mathrm{C}$. The plates were washed with PBST, and $100 \mu \mathrm{l}$ of horseradish peroxidase (HRP)-conjugated anti-pig IgA (AbD Serotec, Raleigh, NC, USA) was added to each well at a dilution of 1:3,000 and incubated at $37^{\circ} \mathrm{C}$ for $1 \mathrm{~h}$. After washing with PBST, 2, 2'-azino-bis (3-ethylbenzthiazoline-6-sulfonic acid) (ABTS) substrate (KPL, Gaithersburg, MD, USA) was added. Finally, the plates were read at an absorbance of $405 \mathrm{~nm}$ using a SpectraMax ELISA reader (Molecular devices, Union City, CA).

For IgG detection, plates were prepared as above, the 4-fold serially diluted sera were incubated for $1 \mathrm{~h}$, and the plates were washed with PBST. Biotinylated anti-pig IgG (KPL, Gaithersburg, MD, USA) was added at $100 \mu \mathrm{l}$ per well at a dilution of 1:5,000 and incubated for $1 \mathrm{~h}$ at $37{ }^{\circ} \mathrm{C}$. The plates were washed, and peroxidase-conjugated streptavidin $(1: 10,000)$ (Roche, Indianapolis, IN, USA) was added and incubated at room temperature for $1 \mathrm{~h}$. Plates were washed again, and ABTS substrate was added. Finally, the plates were read at an absorbance of $405 \mathrm{~nm}$ by using a SpectraMax ELISA reader.

Six controls were included: a coating-buffer-only group; a mock LLC-PK cell lysis diluted with coating buffer group; a negative-serum (serum obtained from the mockinfected pig of the same age and serum samples collected pre-inoculation from all pigs) group; a positive-serum (hyperimmune PDCoV serum OH-DC97) group; a secondary-antibody-free group; and a blocking buffer group. Mock-antigen-coated wells did not show a background reaction. Positive samples were those with an absorbance equal to or greater than the cutoff that was determined as the average value of the absorbance of negative control samples plus three times the standard deviation. The antibody titers were calculated and expressed as the reciprocal of the highest serum dilution that was positive for PDCoV IgA or IgG antibodies.

\section{Complete $\mathrm{S}$ and $\mathrm{N}$ gene sequencing and phylogenetic analysis of TC- and Gn-pig-passaged PDCoV OH- FD22}

The LIC of Gn pigs inoculated with TC-PDCoV OH-FD22 $\mathrm{P} 5, \mathrm{P} 20$, and $\mathrm{P} 40$ were designated $\mathrm{Gn}$ pig OH-FD22 P5 (DC124), Gn pig OH-FD22 P20 (DC175), and Gn pig OHFD22 P40 (DC225), respectively. The complete S and N genes of TC-PDCoV OH-FD22 P5 and P40 as well as the Gn-pig-passaged TC-PDCoV OH-FD22 P5, P20, and P40 in the LIC were amplified, cloned and sequenced, and the sequence data were assembled and subjected to 
phylogenetic analysis according to our previously reported methods [7]. The complete $\mathrm{S}$ and $\mathrm{N}$ genes of TC-PDCoV OH-FD22 P5, P40, Gn pig OH-FD22 P5 (DC124), Gn pig OH-FD22 P20 (DC175), and Gn pig OH-FD22 P40 (DC225) were deposited in GenBank under accession numbers KT945050, KT945051, and KT371500 to KT371507.

\section{Results}

\section{Clinical observations and PDCoV RNA titers in the feces and sera of TC-PDCoV OH-FD22- inoculated Gn pigs}

All Gn pigs that were orally inoculated with TC-PDCoV OH-FD22 developed typical clinical disease, characterized by acute and severe watery diarrhea, vomiting, and mild dehydration. Clinical signs developed at PID 1-2, regardless of the cell culture passage level of the viral strains tested (Table 1). The pigs monitored for long-term clinical signs showed moderate to severe diarrhea for about 6-8 days (Fig. 1A). All inoculated pigs exhibited an onset of clinical disease that was similar or later by 1 day than that after inoculation of Gn pigs with the original PDCoV OH-FD22 (Table 1) [12]. The negative control pigs showed no clinical signs during the study period. The RTPCR tests showed no contamination of the diarrheic fecal samples from the Gn-pig-passaged TC-PDCoV with other detectable enteric viruses (PEDV, TGEV, Rota A/C, and caliciviruses).

The kinetics of fecal RNA shedding are shown in Table 1 and Fig. 1B. As reported [12], the original PDCoV OH-FD22-inoculated pigs showed clinical signs at about PID 1, and fecal viral RNA was detected on PID 1, whereas for the TC-PDCoV OH-FD22-inoculated pigs, fecal viral RNA was detected later on PID 2, and the pigs showed clinical signs at PID 1 to 2. Fecal viral RNA peaked on PID 3 to 5 and then decreased gradually thereafter. Similar to the original PDCoV OH-FD22 inoculation, fecal virus shedding in the TC-PDCoV OH-FD22 P5- or P20-inoculated pigs was still detectable at PID 18 and 20, and then negative at PID 19 and 21, respectively. The TC-PDCoV OH-FD22 P40-inoculated pig had PDCoV-RNA-positive feces until PID 15. The negative control pigs did not shed detectable PDCoV viral RNA in the feces throughout the experiment.

PDCoV RNA was detected in the inoculated pigs' sera by qRT-PCR (Table 1). Only the PID 3 sera of the TCPDCoV OH-FD22-inoculated pigs were PDCoV positive, but the viral RNA titer was very low, with a range of 4.8$5.5 \log _{10} \mathrm{GE} / \mathrm{ml}$. The original PDCoV OH-FD22-inoculated pigs were negative for PDCoV RNA in the sera [12].
No infected pigs had detectable viral RNA prior to inoculation in the prebled serum samples.

\section{Gross and histologic lesions and PDCoV-antigen- positive cells in Gn pigs inoculated with TC-PDCoV OH-FD22}

Two duodenal, 8-10 proximal, middle, and distal jejunal, two ileal, and two cecal/colonic tissues and other major organs were collected and tested for all pigs. By macroscopic examination, inoculated Gn pigs 1 (P5), 3 (P20) and 5 (P40) tested at PID 3 exhibited extensive thin and transparent intestinal walls and accumulation of large amounts of yellowish fluid in the small and large intestinal lumen. The other internal organs appeared normal. The other inoculated pigs, pigs 2 (P5), 4 (P20) and 6 (P40), tested at PID 23-24 or 28, and negative control pigs 7 and 8 did not show gross lesions.

Histologic lesions were limited to villous epithelial cells, but not the crypts, of the small and large intestines, and mainly, the jejunum and ileum, as tested at the early stage of infection at PID 3, but not at PID 23-24 or 28 (Table 2). TCPDCoV OH-FD22 P5-inoculated pig 1 and OH-FD22 P20inoculated pig 3 tested at PID 3 (17 days of age) had diffuse, severe villous atrophy in the jejunum (mean VH:CD ratio \pm SDM, $1.3 \pm 0.2$ for pig 1 and $1.4 \pm 0.2$ for pig 3 ) and ileum, with frequent fusion of atrophied villi and diffuse, mild cytoplasmic vacuolation of enterocytes located at the upper half to the tip of the severely atrophied villi. Other major histologic changes in pigs 1 (P5) and 3 (P20) included a diffuse, mild vacuolation of superficial cecal and colonic epithelial cells. On the other hand, TC-PDCoV OH-FD22 P5-inoculated pig 2 tested at PID 28 (42 days of age) and OH-FD22 P20-inoculated pig 4 tested at PID 24 (38 days of age) had no histologic lesions or evident villous atrophy in the jejunum (mean VH:CD ratio $\pm \mathrm{SDM}, 5.0 \pm 0.8$ for pig 2 and $4.3 \pm 0.6$ for pig 4 ) and ileum, similar to the negative control pig 7 euthanized at 17 days of age (mean jejunal VH:CD ratio \pm SDM, $5.6 \pm 0.5$ ) or negative control pig 8 euthanized at 38 days of age (mean jejunal VH:CD ratio $\pm \mathrm{SDM}, 4.0 \pm 0.2$ ). No villous atrophy or histologic lesions were evident in the remainder of the small intestine, duodenum, and other organs of the inoculated pigs 1-4 and negative control pig 7 at the time points tested.

TC-PDCoV OH-FD22 P40-inoculated pig 5 tested at PID 3 (17 days of age) had diffuse, moderate villous atrophy in the jejunum (mean $\mathrm{VH}: \mathrm{CD}$ ratio $\pm \mathrm{SDM}, 2.7 \pm 0.6$ ) and ileum, with frequent fusion of adjoining atrophied villi and diffuse, mild to moderate cytoplasmic vacuolation of enterocytes lining the epithelium of atrophied jejunal and ileal villi (Fig. 2A). Pig 5 had no histologic lesions in the large intestine. The TC-PDCoV OH-FD22 P40-inoculated pig 6 tested at PID 23 (37 days of age) had no histologic 


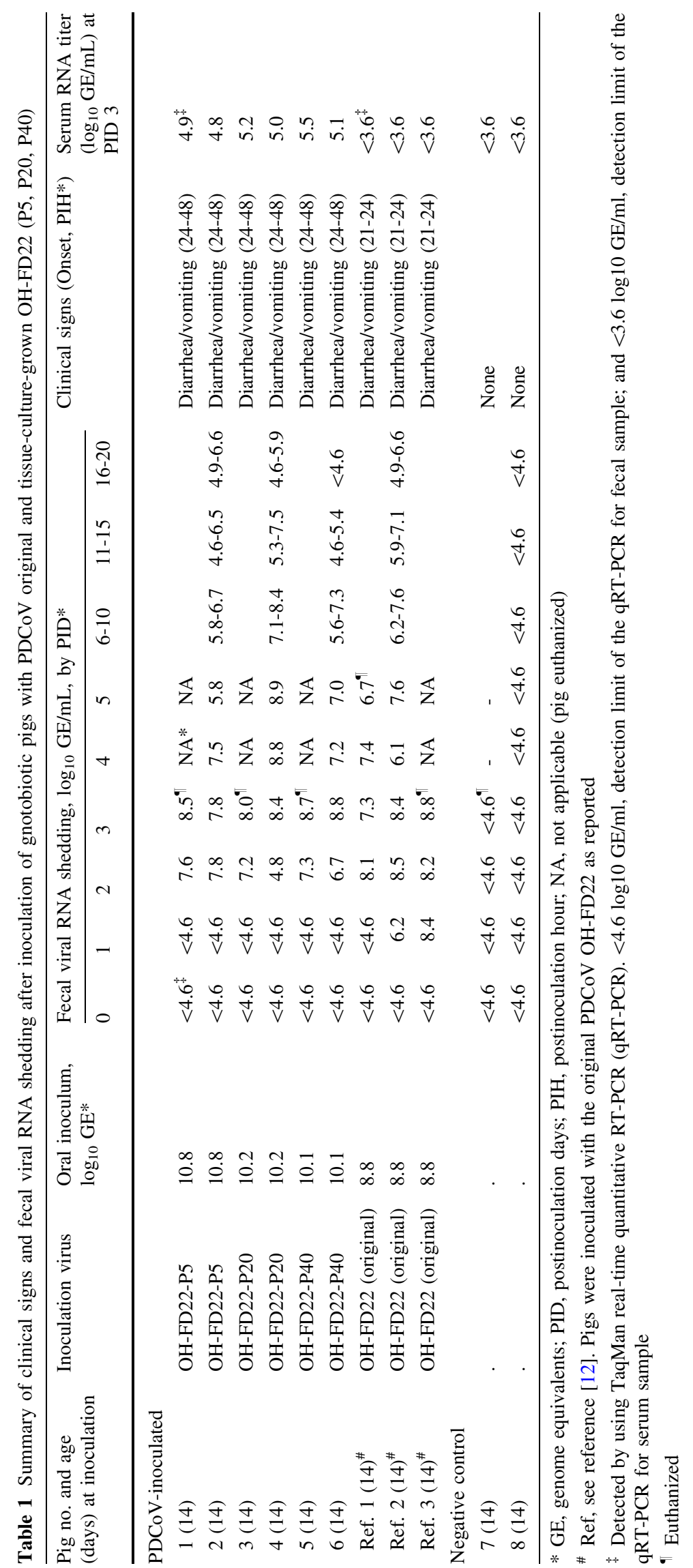


Fig. 1 Diarrhea scores (A) and prolonged fecal viral RNA shedding (B) of Gn pigs inoculated with TC-PDCoV OH-FD22. Fourteen-day-old Gn pigs were inoculated orally with 10.1-10.8 $\log _{10} \mathrm{GEs} / \mathrm{ml}$ of TCPDCoV strains OH-FD22 P5, $\mathrm{P} 20$, and P40. Pigs ( $\mathrm{n}=1$ for each passage) were monitored for long-term clinical signs and fecal viral RNA shedding.

(A) Clinical signs were monitored daily, and diarrhea (fecal consistency) was scored for each pig as follows:

$0=$ solid; $1=$ pasty;

$2=$ semi-liquid; $3=$ liquid, with scores of 2 or more considered diarrheic. (B) Rectal swabs were collected daily from each animal throughout the experiment. The PDCoV fecal RNA shedding titers were determined by qRT-PCR

\section{A Diarrhea scores in gnotobiotic pigs inoculated with the original and cell culture PDCoV OH-FD22 strains}

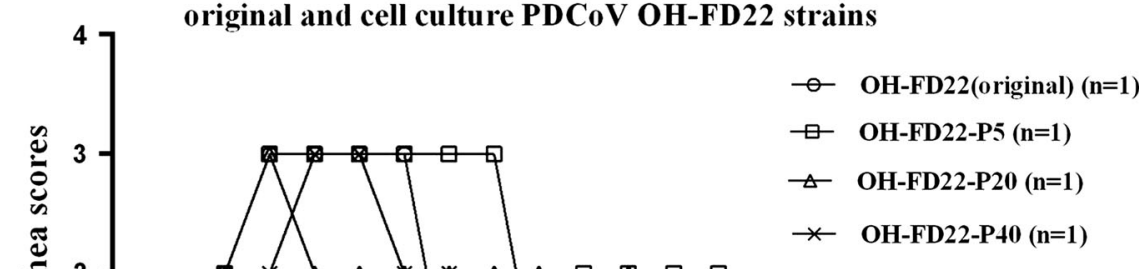

B PDCoV fecal RNA shedding in gnotobiotic pigs inoculated the with original and cell culture PDCoV OH-FD22 strains

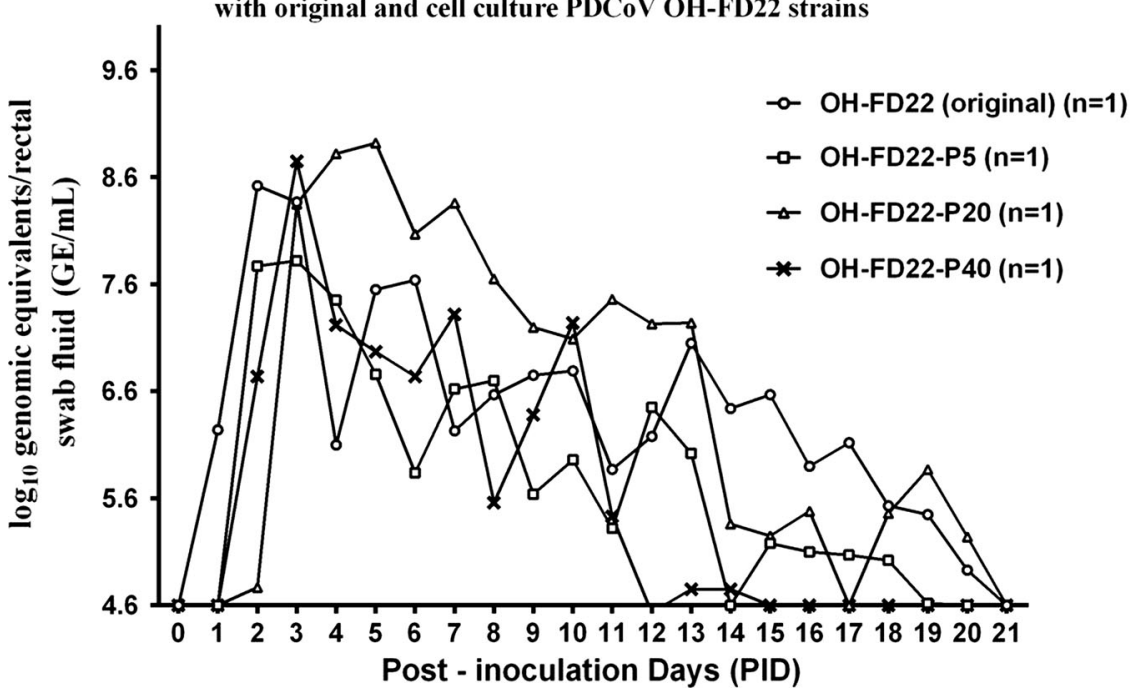

lesions or villous atrophy in the jejunum (mean VH:CD ratio $\pm \mathrm{SDM}, 3.4 \pm 0.8$ ) and ileum compared to negative control pig 8 euthanized at 38 days of age (mean jejunal $\mathrm{VH}: \mathrm{CD}$ ratio $\pm \mathrm{SDM}, 4.0 \pm 0.2$ ) (Table 2). No villous atrophy or other histologic lesions were evident in the remainder of the small intestine, duodenum, and other organs of inoculated pigs 5 and 6 and negative control pig 8 .

As determined using formalin-fixed, paraffin-embedded tissues, IF-stained cells were observed mainly in the atrophied villous epithelium of the small intestine (Fig. 2B), proximal jejunum to ileum, and occasionally, in the duodenum and cecum/colon of pigs 1 (P5), 3 (P20) and 5 (P40) tested at PID 3 (Table 2), as reported previously [4, 12]. The fluorescence was confined to the cytoplasm of the villous epithelial cells. Pigs 1 (P5), 3 (P20) and 5 (P40) also had a few IF-stained cells in the mesenteric lymph nodes. No other internal organs of the infected pigs tested at PID 3 showed IFpositive staining. When tested at PID 23-24, no IF-stained cells were detected in the villous epithelium of the small or large intestine of inoculated pigs 4 (P20) and 6 (P40). However, small numbers of IF-stained cells were observed in the lamina propria of the villi and crypts of the proximal jejunum to ileum (Fig. 2C), and to a lesser extent, in duodenum and cecum/colon. Moderate numbers of IF-stained cells were also detected in Peyer's patches (Fig. 2C). Pigs 4 (P20) and 6 (P40) also had small to moderate numbers of IFstained cells in the mesenteric lymph nodes (Fig. 2D); however, no other internal organs of infected pigs showed IF-positive staining. No IF-stained cells were detected in the negative control pigs 7 and 8 (Fig. 2E and F).

\section{Serum antibody responses to PDCoV}

Serum samples were collected at PID 1, 7, 14, and 23/24 to test for PDCoV-specific IgG and IgA antibodies by indirect ELISA (Table 3). All inoculated pigs developed PDCoV- 


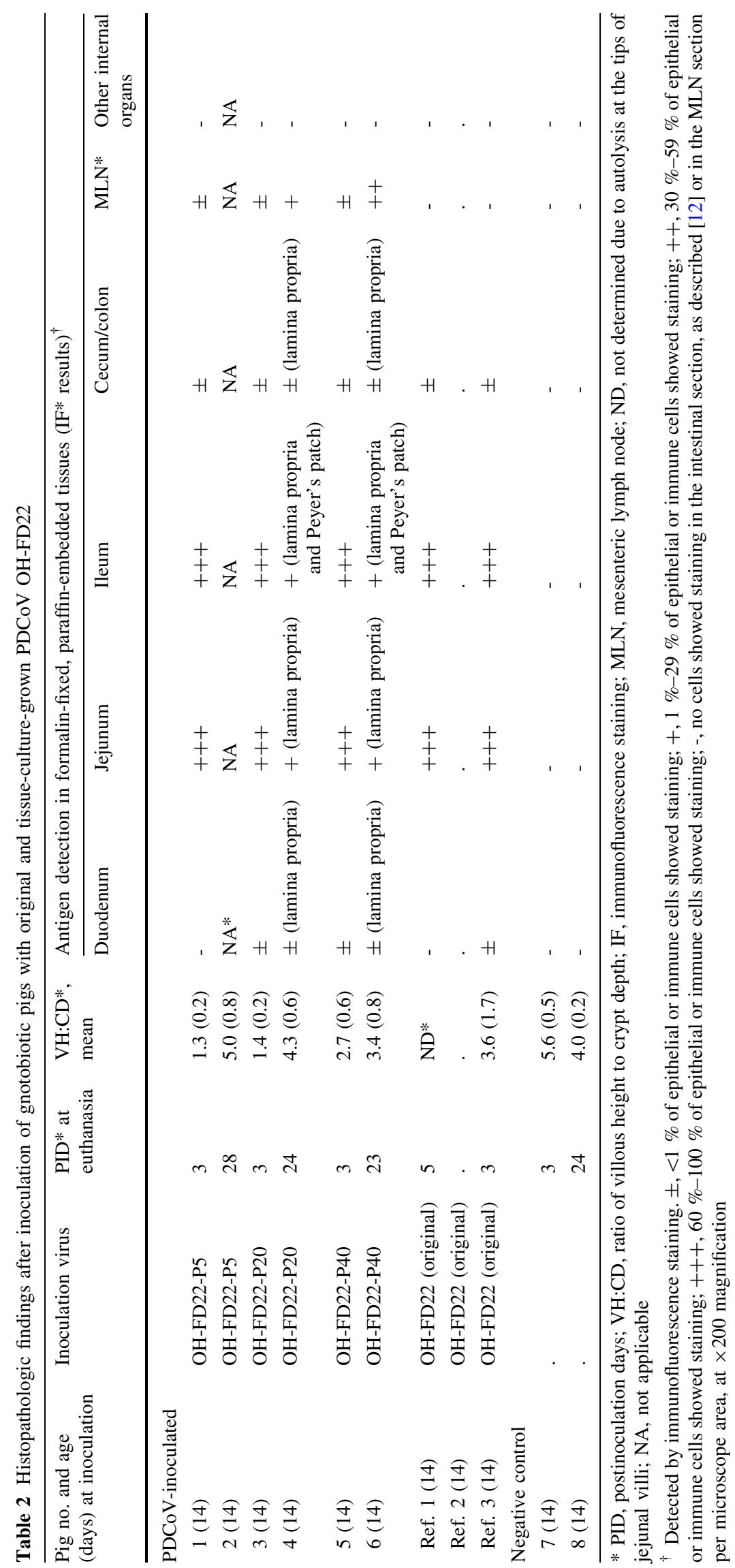



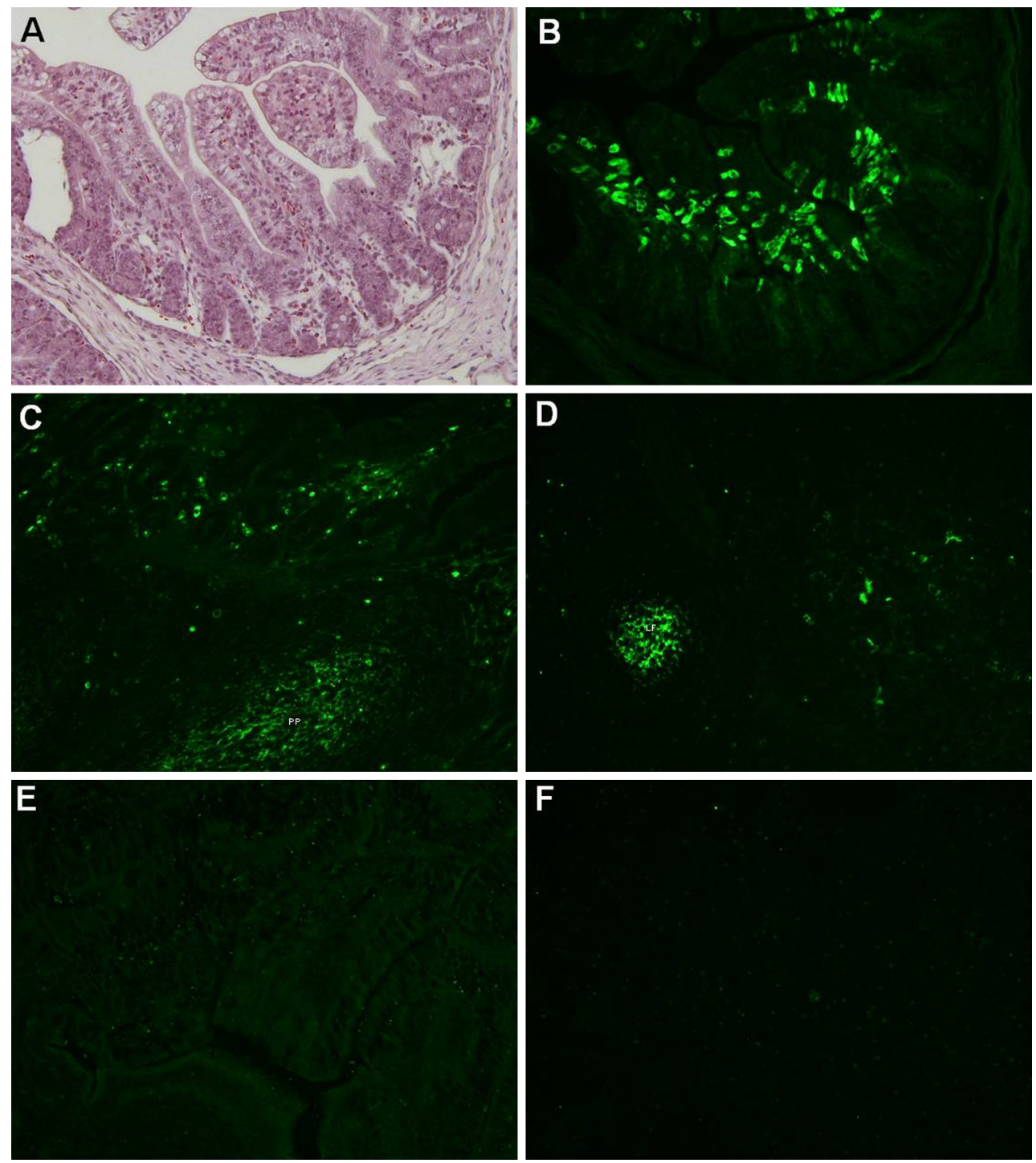

Fig. 2 Histopathology and localization of PDCoV antigens by immunofluorescence (IF) staining in the small intestine and mesenteric lymph node of gnotobiotic pigs inoculated with TC-PDCoV OHFD22. Pigs were inoculated with TC-PDCoV OH-FD22 $(n=2$ for each passage). One pig in each group was euthanized for pathologic examination at PID 3, and the remainder were euthanized at PID 23-24 or 28. (A) Hematoxylin and eosin-stained jejunum of inoculated pig 5 at post-inoculation day (PID) 3, showing diffuse, moderate atrophic enteritis, with diffuse, mild vacuolation of enterocytes lining the epithelium of atrophied villi. (B) IF staining of the jejunum of inoculated pig 5 at PID 3, showing PDCoV antigen in the epithelial

specific IgG antibodies at PID 14, with IgG antibody titers of 16 for the TC-PDCoV OH-FD22 P5 and P20 groups, and of 64-256 for the TC-PDCoV OH-FD22 P40 and the original inoculated pigs. At PID 23, the TC-PDCoV OHFD22 P5, P40 and the original inoculated pigs had the highest IgG antibody titers (1024), while the TC-PDCoV OH-FD22 P20 induced the lowest IgG antibody titer of 256. The negative control pig was negative for PDCoVspecific IgG antibodies at all experimental time points.

cells lining the atrophied villi. (C) IF staining of ileum of inoculated pig 6 at PID 23, showing small to moderate numbers of IF-stained cells in the lamina propria of villi and crypts and Peyer's patches. Note that no IF-stained cells were evident in the villous epithelium. (D) IF staining of mesenteric lymph node of inoculated pig 6 at PID 23 , showing moderate numbers of IF-stained cells in the lymphoid follicle. (E) IF staining of ileum of uninoculated, negative control pig 8 at PID 24 (38 days of age), showing no IF-stained cells in the lamina propria of villi and crypts or Peyer's patches. (F) IF staining of mesenteric lymph node of the uninoculated, negative control pig 8 at PID 24, showing no IF-stained cells. Original magnification, all $\times 200$

PDCoV-specific IgA antibodies in sera were also determined by indirect ELISA (Table 3). Titers of IgA antibodies were detected at PID 14 in all the inoculated pigs' sera, with a lower IgA antibody titer of 16 for the TCPDCoV OH-FD22 P5 and P20 groups, and higher (64) for the TC-PDCoV OH-FD22 P40 and the original inoculated pigs. The highest IgA antibody titers were detected at PID 23/24. TC-PDCoV OH-FD22 P40 elicited the highest IgA antibody titer (1024) at PID 23, whereas TC-PDCoV OH- 
Table 3 Detection of PDCoV-specific IgA, IgG and neutralizing antibody titers in the sera of Gn pigs inoculated with original and tissueculture-grown PDCoV OH-FD22

\begin{tabular}{|c|c|c|c|c|c|c|c|c|c|c|c|c|c|}
\hline \multirow[t]{2}{*}{ Pig no. } & \multirow[t]{2}{*}{ Inoculation virus } & \multicolumn{3}{|c|}{ PID* 1} & \multicolumn{3}{|c|}{ PID 7} & \multicolumn{3}{|c|}{ PID 14} & \multicolumn{3}{|c|}{ PID 23/24 } \\
\hline & & $\operatorname{Ig} \mathrm{A}$ & $\operatorname{IgG}$ & $\mathrm{VN}^{*}$ & $\operatorname{IgA}$ & $\operatorname{IgG}$ & VN & $\operatorname{Ig} \mathrm{A}$ & $\mathrm{IgG}$ & VN & $\operatorname{IgA}$ & $\mathrm{IgG}$ & VN \\
\hline 2 & OH-FD22-P5 & $<4$ & $<4$ & $<4$ & $<4$ & $<4$ & 64 & 16 & 16 & 256 & 256 & 1024 & 1024 \\
\hline 4 & OH-FD22-P20 & $<4$ & $<4$ & $<4$ & $<4$ & $<4$ & 64 & 16 & 16 & 256 & 256 & 256 & 1024 \\
\hline 6 & OH-FD22-P40 & $<4$ & $<4$ & $<4$ & $<4$ & $<4$ & 256 & 64 & 64 & 1024 & 1024 & 1024 & 4096 \\
\hline $2^{\#}$ & OH-FD22 (original) & $<4$ & $<4$ & $<4$ & $<4$ & $<4$ & 64 & 64 & 256 & 256 & 256 & 1024 & 1024 \\
\hline 8 & - & $<4$ & $<4$ & $<4$ & $<4$ & $<4$ & $<4$ & $<4$ & $<4$ & $<4$ & $<4$ & $<4$ & $<4$ \\
\hline
\end{tabular}

* PID, postinoculation days; VN, virus neutralization

\# See reference [12]. Pig 2 was inoculated with the original PDCoV OH-FD22 as reported

Note: PDCoV-specific IgG and IgA antibodies in serum were determined by indirect ELISA using cell culture PDCoV OH-FD22 as the coating antigen. The neutralizing antibody titers were tested by the $80 \%$ plaque reduction neutralization test $\left(\mathrm{PRNT}_{80}\right) . \mathrm{PRNT}_{80} \mathrm{Was}_{\mathrm{W}}$ determined as the reciprocal of the highest serum dilution that neutralized $80 \%$ of the plaques as compared to that in the virus-only control wells. The IgA, IgG and VN antibody titers of hyperimmune PDCoV serum OH-DC97 (positive control serum) were 1024, 4096 and 4096, respectively

FD22 P5, P20 and the original PDCoV OH-FD22 induced IgA antibody titers of 256 at PID 24. The negative control pig was negative for PDCoV-specific IgA antibodies at all experimental times.

\section{PDCoV-neutralizing antibodies}

To investigate if PDCoV-neutralizing antibodies were induced, a plaque reduction PDCoV neutralization assay $\left(\mathrm{PRNT}_{80}\right)$ was performed. Serum samples at PID 1, 7, 14, and 23/24 were tested for PDCoV neutralizing antibody titers (Table 3). All inoculated pigs tested at the later stage of infection (PID 23/24) exhibited high PDCoVneutralizing titers. Regardless of the cell culture passage number of the virus strains used, serum virus neutralization (VN) antibodies were first detected in all of the original and TC-PDCoV OH-FD22-inoculated pigs at PID 7 (64-256), and thereafter, the titers increased gradually and peaked at PID 23/24. TC-PDCoV strain OH-FD22 $\mathrm{P} 40$ induced the highest VN titer at the time points tested (PID 7, 14 and 23), which was 4 times higher than that of the other groups. There were no differences in VN antibody titers among the Gn pigs inoculated with the TCPDCoV strains OH-FD22 P5 and P20, and original PDCoV OH-FD22. No neutralizing antibodies were observed for the control pig serum.

\section{Phylogenetic analysis of the $\mathrm{S}$ and $\mathrm{N}$ genes of TC- and Gn pig-passaged PDCoV OH-FD22}

To examine if genetic changes occurred in the major structural genes of PDCoV OH-FD22 during serial passages in LLC-PK cells and Gn pigs, the complete $\mathrm{S}$ and $\mathrm{N}$ genes of TC- and Gn- pig-passaged PDCoV OH-FD22 P5, P20 and P40 were sequenced and compared with those of the original PDCoV OH-FD22. The S genes of Gn-pig- passaged PDCoV OH-FD22 P5, P20 and P40 strains shared $99.8 \%$ to $100 \%$ nucleotide sequence identity, and they shared $99.7 \%$ to $100 \%$ nucleotide sequence identity with the original PDCoV OH-FD22 and TC-PDCoV OHFD22 (P5, P11, P20 and P40). All of the PDCoV OHFD22-related S genes shared $98.4 \%$ to $100 \%$ nucleotide sequence identity with other PDCoV strains available in GenBank (Fig. 3). When comparing the TC-PDCoV OHFD22 P5 strain with the original OH-FD22 strain, only one nucleotide change was found in the $\mathrm{S}$ gene at position $466 \mathrm{nt}$ (nucleotides and amino acids are numbered according to the $\mathrm{S}$ gene of the PDCoV OH-FD22 sequence [GenBank accession no. KP995358]), which resulted in an amino acid (aa) change (Val changed to Phe at position 163 aa). This was subsequently found in the following sequenced TC-PDCoV OH-FD22 passages (P20 and P40) [7] (Table 4). The TC-PDCoV OH-FD22 P11 (passage 11 in LLC-PK cells) and P20 had five nucleotide changes (at positions 430, 466, 1191, 2456, and $3331 \mathrm{nt}$ ) in the $\mathrm{S}$ gene when compared to the original OH-FD22 strain, which also resulted in amino acid changes [7]. In the current study, the $\mathrm{S}$ genes of $\mathrm{TC}$ PDCoV OH-FD22 P40, pig-passaged OH-FD22 P20 and P40 had the same changes (Table 4). When comparing the S gene of TC-PDCoV OH-FD22 P40 with the original virus and TC-PDCoV OH-FD22 (P5 and P20), one nucleotide change was found in the $\mathrm{S}$ gene at position 991 nt, which resulted in an aa change (Asn changed to Asp at position 331 aa). The Gn-pig passaged TC-PDCoV OHFD22 P40 (DC225) had the same change in this position (Table 4). Only one nucleotide change was found in the $\mathrm{S}$ gene of pig-passaged PDCoV (OH-FD22 P20 [DC175] and $\mathrm{OH}-\mathrm{FD} 22 \mathrm{P} 40$ [DC225]) when compared with the corresponding TC-PDCoV OH-FD22 P20 and P40 at position 2,842 nt of the $\mathrm{S}$ gene. This nucleotide change resulted in an amino acid change (Ala changed to Thr at 
Fig. 3 Phylogenetic analysis of complete $\mathrm{S}$ and $\mathrm{N}$ gene nucleotide sequences of TCand Gn-pig-passaged PDCoV OH-FD22 (indicated by triangles) and other published PDCoV sequences. Reference sequences obtained from GenBank are indicated by strain names and accession numbers (in parentheses). The trees were constructed by using the maximum-likelihood method with MEGA6.06 software (http://www.megasoftware.net/ ). Bootstrap analysis was carried out on 1,000 replicate data sets, and values are indicated adjacent to the branching points. Bars represent 0.001 nucleotide substitutions per site

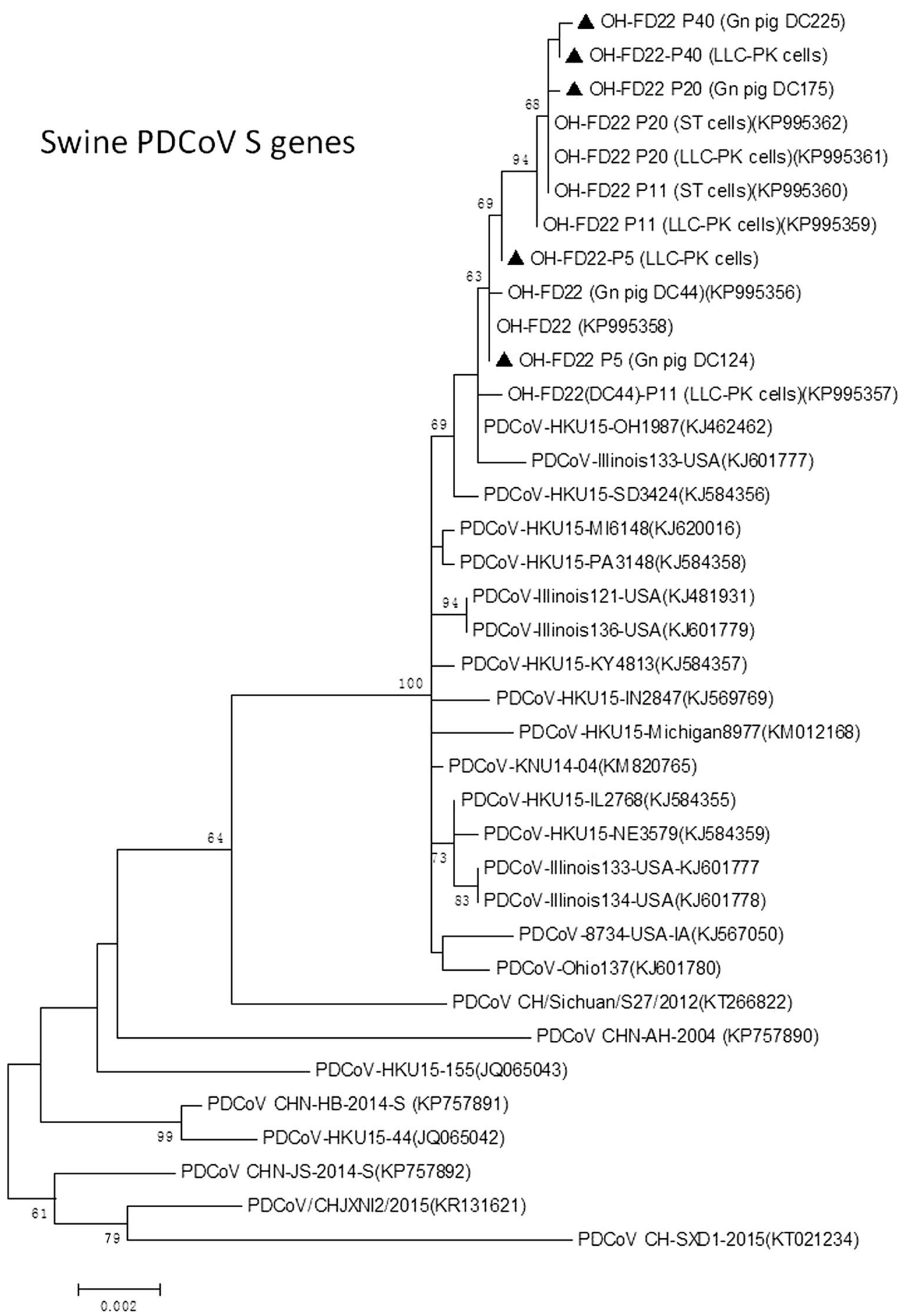

position 948) (Table 4). Phylogenetic analysis of the $\mathrm{S}$ genes showed that all of the US PDCoV strains (including the OH-FD22 strains) clustered in one group, which had a more distant relationship to the Hong Kong PDCoV strains (HKU15-44 and HKU15-155) and the mainland China strains. Furthermore, all PDCoV OH-FD22 strains clustered into a subclade, while other US PDCoV strains were clustered separately (Fig. 3).
The $\mathrm{N}$ genes of the pig-passaged and the corresponding TC-PDCoV OH-FD22 shared $100 \%$ nucleotide sequence identity and were also $100 \%$ identical to the original PDCoV OH-FD22 N gene. They shared $98.9 \%$ to $99.9 \%$ nucleotide sequence identity with the other PDCoV strains available in GenBank. Phylogenetic analysis of the $\mathrm{N}$ genes of the pigpassaged and corresponding TC-PDCoV OH-FD22 revealed that they belonged to the same group (Fig. 3). 
Fig. 3 continued

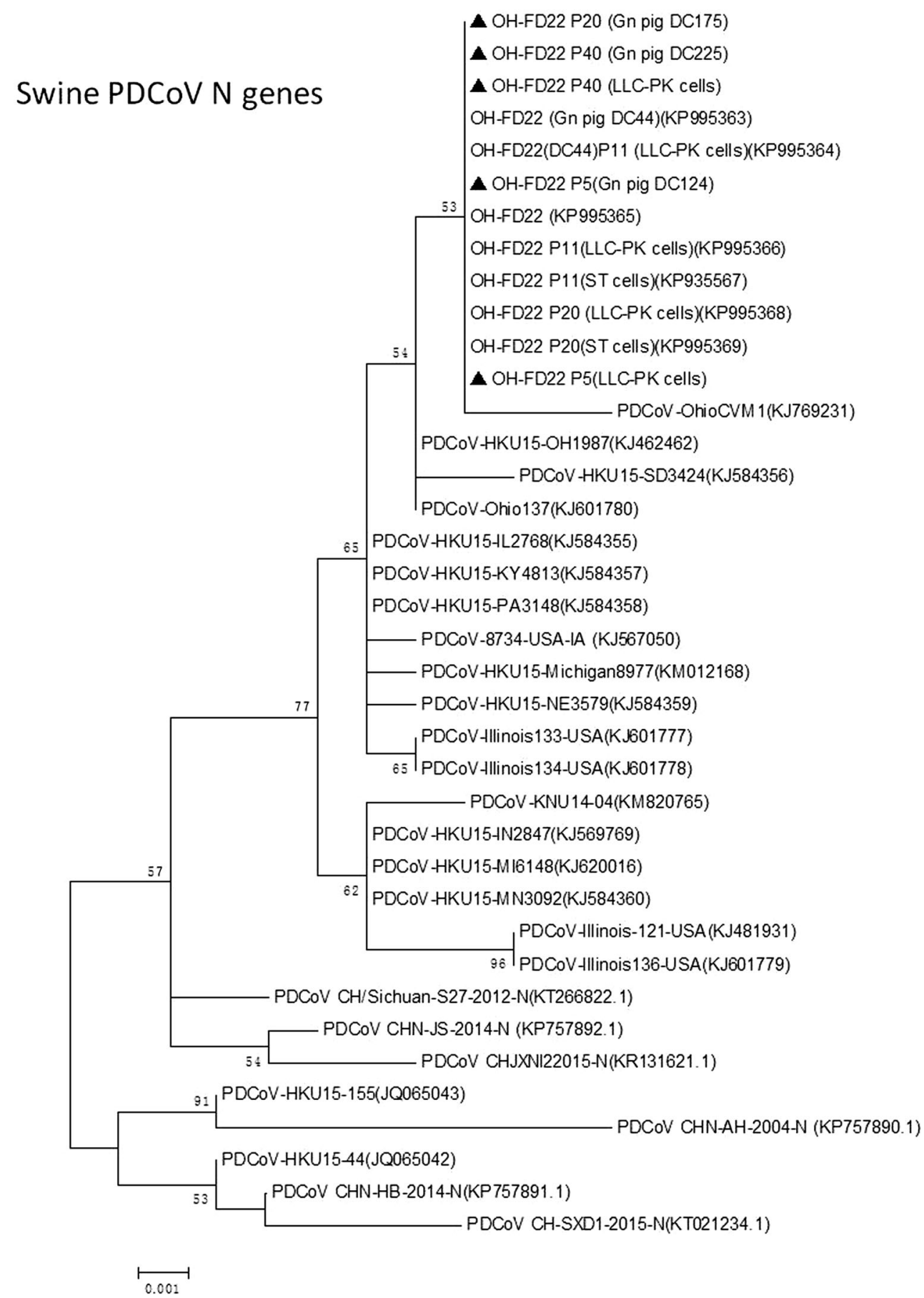

\section{Discussion}

Our study demonstrated similar clinical diseases and pathologic lesions in all 14-day-old Gn pigs inoculated with the TC-PDCoV OH-FD22 P5, P20, and P40 strains when compared with previous observations in Gn pigs of the same age inoculated orally with the original field PDCoV OH-FD22 [12]. However, the following differences were identified: i) earlier onset of clinical signs and fecal virus shedding by PID 1 in original virus-inoculated pigs (Table 1) [12]; ii) frequent detection $(6 / 6 ; 100 \%)$ of transient viremia (viral RNA) in serum in TC-PDCoV OHFD22-inoculated pigs on PID 3, while no viremia was detected in the original virus-inoculated pigs at PID 3 and other time points (Table 1) [12]; and iii) in addition to $\mathrm{PDCoV}$ antigen-positive enterocytes in the small and large intestines at the early stage of infection (Fig. 2B), a few to moderate numbers of PDCoV-antigen-positive cells were also detected by IF in the intestinal lamina propria and mesenteric lymph nodes of the TC-PDCoV OH-FD22- 
Table 4 Nucleotide and amino acid changes in the S gene/protein of PDCoV OH-FD22 passaged in cell culture and in Gn pigs

\begin{tabular}{|c|c|c|c|c|c|c|c|}
\hline Virus Position* & $\begin{array}{l}430 \mathrm{nt}^{* *} \\
\left(\mathrm{aa}^{* *} 144\right)\end{array}$ & $\begin{array}{c}466 \mathrm{nt} \\
\text { (aa 156) }\end{array}$ & $\begin{array}{l}991 \mathrm{nt} \\
\text { (aa 331) }\end{array}$ & $\begin{array}{l}1191 \mathrm{nt} \\
\text { (aa 397) }\end{array}$ & $\begin{array}{l}2456 \mathrm{nt} \\
\text { (aa } 819)\end{array}$ & $\begin{array}{l}2842 \mathrm{nt} \\
\text { (aa 948) }\end{array}$ & $\begin{array}{r}3331 \mathrm{nt} \\
\text { (aa 1111) }\end{array}$ \\
\hline PDCoV OH-FD22 (original) ${ }^{\#}$ & G (Glu) & $\mathrm{G}$ (Val) & $\mathrm{A}(\mathrm{Asn})$ & $\mathrm{T}$ (Asn) & $\mathrm{C}(\mathrm{Thr})$ & G (Ala) & G (Ala) \\
\hline $\begin{array}{l}\text { Gn pig-passaged PDCoV OH- } \\
\text { FD22 (DC44) }\end{array}$ & G (Glu) & $\mathrm{G}$ (Val) & A (Asn) & $\mathrm{T}$ (Asn) & $\mathrm{C}$ (Thr) & G (Ala) & G (Ala) \\
\hline TC-PDCoV OH-FD22 P5 & G (Glu) & $\mathrm{T}$ (Phe) & A (Asn) & $\mathrm{T}$ (Asn) & C (Thr) & G (Ala) & G (Ala) \\
\hline $\begin{array}{l}\text { Gn pig-passaged TC-PDCoV } \\
\text { OH-FD22 P5 (DC124) }\end{array}$ & G (Glu) & $\mathrm{T}$ (Phe) & A (Asn) & $\mathrm{T}$ (Asn) & $\mathrm{C}(\mathrm{Thr})$ & G (Ala) & G (Ala) \\
\hline TC-PDCoV OH-FD22 P20 \# & $\mathrm{C}(\mathrm{Gln})$ & $\mathrm{T}$ (Phe) & $\mathrm{A}(\mathrm{Asn})$ & A (Lys) & $\mathrm{T}$ (Ile) & G (Ala) & A (Thr) \\
\hline $\begin{array}{l}\text { Gn pig-passaged TC-PDCoV } \\
\text { OH-FD22 P20 (DC175) }\end{array}$ & $\mathrm{C}(\mathrm{Gln})$ & $\mathrm{T}(\mathrm{Phe})$ & A (Asn) & A (Lys) & $\mathrm{T}$ (Ile) & A (Thr) & A (Thr) \\
\hline TC-PDCoV OH-FD22 P40 & $\mathrm{C}(\mathrm{Gln})$ & $\mathrm{T}$ (Phe) & $\mathrm{G}$ (Asp) & A (Lys) & $\mathrm{T}$ (Ile) & G (Ala) & $\mathrm{A}(\mathrm{Thr})$ \\
\hline $\begin{array}{l}\text { Gn pig-passaged TC-PDCoV } \\
\text { OH-FD22 P40 (DC225) }\end{array}$ & $\mathrm{C}(\mathrm{Gln})$ & $\mathrm{T}$ (Phe) & $\mathrm{G}$ (Asp) & A (Lys) & $\mathrm{T}$ (Ile) & A (Thr) & A (Thr) \\
\hline
\end{tabular}

* nucleotides and amino acids are numbered according to the S gene of the PDCoV OH-FD22 sequence (GenBank accession no. KP995358) [7]

** nt, nucleotide; aa, amino acid

\# See reference [7]

inoculated pigs when tested at PID 3 and PID 23-24 (Fig. 2C and D; Table 2). In this study, no attenuation of the TC-PDCoV OH-FD22 P40 strain (cell passage 40) in pigs was evident, since severe clinical disease and lesions were reproduced in the two inoculated Gn pigs. Further studies are needed to investigate if higher levels of cellculture passaged TC-PDCoV OH-FD22 are attenuated.

In our study, 14-day-old Gn pigs inoculated with 10.1$10.8 \log _{10} \mathrm{GE}\left(\approx 9 \log _{10}\right.$ PFU per pig [7]) of TC-PDCoV OH-FD22 P5, P20, and P40 all (6/6, $100 \%)$ showed severe diarrhea and/or vomiting at PID 2, which coincided with the first detection of viral RNA in feces (Fig. 1B and Table 1). Fecal PDCoV shedding titers peaked at PID 3 to 5 (Fig. 1B and Table 1). Another study using conventional 5-day-old pigs and a cell-culture-adapted PDCoV USA/IL/ 2014 strain (passage 11) reported onset of mild diarrhea (soft feces) at PID 5 in five pigs that were orally inoculated with $3 \times 10^{4} \mathrm{TCID}_{50}$ of the virus, which was later than or coincided with the first detection of viral RNA in feces at PID 4 (3/5 pigs tested) or 5 (2/5 pigs tested) [4]. In another study of a cell-culture-grown PDCoV Michigan/8977/2014 strain and an Ohio wild-type field CVM1 strain using 10-day-old conventional or Gn pigs, moderate to severe diarrhea occurred in pigs orally inoculated with $10^{6}$ genomic RNA copies or PFU/pig of each strain at PID 1-3. The Gn pigs inoculated with the CVM1 field strain exhibited more-severe clinical disease or histologic lesions compared to $\mathrm{Gn}$ or conventional pigs inoculated with the cell-culture-adapted Michigan/8977/2014 strain (cell passage 15) [17]. These discrepant observations may be due to: i) differences in the PDCoV strains used, inoculation doses, and cell passage level; ii) conventional vs. Gn pigs used and different ages of pigs used; and iii) selective pig euthanization that could influence fecal virus shedding titers in the remaining pigs at the later stages of infection. The two strains, USA/IL/2014 (GenBank accession no. KP981395) and Michigan/8977/2014 (GenBank accession no. KM012168), shared $100 \%$ identical sequences for the complete $\mathrm{S}$ gene. The $\mathrm{S}$ gene of the original $\mathrm{PDCoV} \mathrm{OH-}$ FD22 strain (GenBank accession no. KP995358) had $99.7 \%$ nucleotide sequence identity with that of the USA/ IL/2014 and Michigan/8977/2014 strain.

In our study, we detected PDCoV RNA in the feces of TC-PDCoV OH-FD22 P5 or P20-inoculated Gn pigs collected until PID 18-20, indicating prolonged fecal virus RNA shedding in PDCoV-infected pigs. However, a shorter duration of fecal viral RNA shedding by 3-5 days was noted in a TC-PDCoV OH-FD22 P40-inoculated pig that shed until PID 15 (Fig. 1B). Since the pig exhibited moderate to severe clinical signs and gross and histologic lesions (intestinal villous atrophy) and high serum antibody titers to PDCoV, based on a single pig, it is unclear if the shorter duration of fecal RNA virus shedding is related to any reduced virulence of TC-PDCoV OH-FD22 P40. Higher passage levels of the TC-PDCoV OH-FD22 strain should be tested for attenuation in additional pigs in a future study. In addition, low PDCoV RNA titers were detected in sera collected in the acute stage from the TCPDCoV OH-FD22 inoculated pigs at PID 3, which coincided with previous studies, showing frequent detection of 
PDCoV RNA in serum collected at early stages of infection [4, 17], although sera from the parental PDCoV OH-FD22inoculated pigs were PDCoV RNA negative at PIDs 3-7 [12]. In the previous study, the infectious titer of the wildtype PDCoV in the fecal sample was unknown because it does not grow in tissue culture. However, the viral RNA titer of the wild-type PDCoV OH-FD22 was determined by qRT-PCR. Three 14-day-old Gn pigs were inoculated orally with $8.8 \log _{10}$ GE of the virus [12], which was approximately $2 \log _{10}$ GE lower than that of the inoculum of TC-PDCoV OH-FD22 used in the current study. Based on titer differences in the virus inocula, we speculate that the lower titer of the inoculation dose might be an explanation for lack of detection of viremia (viral RNA) in the wild-type PDCoV OH-FD22 inoculated pigs.

Relative to PDCoV infection, higher titers of PEDV RNA in serum were detected during the acute stage of infection $[11,18]$. Further studies are needed to determine whether PDCoV present in serum remains infectious in pigs.

The severity of clinical disease and histologic lesions (intestinal villous atrophy) in all TC-PDCoV OH-FD22 P5, P20, and P40-inoculated Gn pigs was similar to that in Gn pigs inoculated with the original field PDCoV OH-FD22 in our previous study conducted under similar experimental conditions [12]. At the early stage of infection (PID 3), the majority of PDCoV antigen-positive cells were in intestinal villous epithelial cells in jejunum and ileum. A few macrophage-like cells located in the lamina propria and Peyer's patches of the jejunum and ileum were also positive for PDCoV antigen by IF. In contrast, after recovery at PID 23-24, when no fecal virus RNA shedding or clinical disease was detected, moderate to large numbers of PDCoVantigen-positive cells were found by IF in the intestinal lamina propria, Peyer's patches and mesenteric lymph nodes, in what appeared to be macrophage-like cells. Because there was no detectable fecal virus RNA shedding at this time, PDCoV antigens on the surface or within the cells may not indicate replicating virus, but possibly antigen that was taken up.

In PEDV-infected pigs, serum PEDV-specific antibody was first detected at about PID 7 to $10[5,18]$. There is no information on the development of PDCoV-specific antibodies in serum of PDCoV-infected pigs, and to our knowledge this is the first report of development of an assay for PDCoV serology. In our study, 14-day-old inoculated $\mathrm{Gn}$ pigs had detectable serum $\mathrm{IgG} / \mathrm{IgA}$ antibodies at PID 14 and VN antibodies at PID 7 (Table 3). Thereafter, PDCoV-specific IgG, IgA and VN antibody titers increased and remained high at the end of the experiment at PID 23/24, when the pigs were fully recovered from clinical disease and fecal virus RNA shedding. Compared to TC-PDCoV OH-FD22 P5- or P20-inoculated pigs, the TC-PDCoV OH-FD22 P40-inoculated pig had higher serum PDCoV-specific VN antibody titers at the time points tested. Because of the limited number of pigs tested, it is unclear whether these observations were related to the shorter duration (by 5 days) of fecal shedding of virus RNA.

CoVs have high mutation rates and can easily undergo recombination and deletion events, leading to altered tissue tropism, transmission routes, and host specificity [17]. In our study, a comparative analysis of the sequenced $\mathrm{S}$ genes of TC- and Gn-pig-passaged TC-PDCoV OH-FD22 showed that only one nucleotide change occurred in the $\mathrm{S}$ gene of the Gn-pig-passaged TC-PDCoV OH-FD22 P20 (DC175) and P40 (DC225) when compared with the corresponding TC-PDCoV OH-FD22 P20 and P40. Because TC-PDCoV OH-FD22 P20 or P40-inoculated pigs showed severe clinical disease and histologic lesions, the single nucleotide change likely does not reflect changes related to the pathogenicity of TC-PDCoV OH-FD22. However, a nucleotide change in the TC-PDCoV OH-FD22 P5 and five subsequent nucleotide changes in the TC-PDCoV OHFD22 P11 and the conservation of these changes in passages (P20 and P40) might reflect changes related to adaptation of PDCoV to cell culture.

In conclusion, our study reproduced and confirmed the enteropathogenicity of TC-PDCoV OH-FD22 P5, P20 and P40 strains. The virulence appeared to be similar to that of the original field PDCoV OH-FD22 strain, as evident by severe diarrhea/vomiting, atrophic enteritis, and high levels of serum IgG, IgA and VN antibodies in the Gn pigs. These findings suggest that the TC-PDCoV OH-FD22 strains tested in this study will be useful for PDCoV pathogenesis studies and evaluation of higher-cell-culture passaged TCPDCoV OH-FD22 strains to verify attenuation and vaccine potential.

\section{Compliance with ethical standards}

Funding This research was supported by state and federal funds appropriated to the Ohio Agricultural Research and Development Center, The Ohio State University, and Four Star Animal Health Inc. (Loramie, OH, USA) (L.J. Saif, principal investigator).

Ethical approval All applicable international, national, and/or institutional guidelines for the care and use of animals were followed.

Conflict of interest All authors declare that there are no financial or other relationships that might lead to a conflict of interest. All authors have seen and approved the manuscript.

\section{References}

1. Amimo JO, Okoth E, Junga JO, Ogara WO, Njahira MN, Wang Q, Vlasova AN, Saif LJ, Djikeng A (2013) Molecular detection and genetic characterization of kobuviruses and astroviruses in 
asymptomatic local pigs in East Africa. Arch Virol 159:1313-1319

2. Amimo JO, Vlasova AN, Saif LJ (2013) Prevalence and genetic heterogeneity of porcine group $\mathrm{C}$ rotaviruses in nursing and weaned piglets in Ohio, USA and identification of a potential new VP4 genotype. Vet Microbiol 164:27-38

3. Bradford MM (1976) A rapid and sensitive method for the quantitation of microgram quantities of protein utilizing the principle of protein-dye binding. Anal Biochem 72:248-254

4. Chen Q, Gauger P, Stafne M, Thomas J, Arruda P, Burrough E, Madson D, Brodie J, Magstadt D, Derscheid R, Welch M, Zhang J (2015) Pathogenicity and pathogenesis of a United States porcine deltacoronavirus cell culture isolate in 5-day-old neonatal piglets. Virology 482:51-59

5. de Arriba CA, Pozo J, Rubio P (2002) Mucosal and systemic isotype-specific antibody responses and protection in conventional pigs exposed to virulent or attenuated porcine epidemic diarrhoea virus. Vet Immunol Immunopathol 85:85-97

6. Dong N, Fang L, Zeng S, Sun Q, Chen H, Xiao S (2015) Porcine deltacoronavirus in Mainland China. Emerg Infect Dis 21:2254-2255

7. Hu H, Jung K, Vlasova AN, Chepngeno J, Lu Z, Wang Q, Saif LJ (2015) Isolation and characterization of porcine deltacoronavirus from pigs with diarrhea in the United States. J Clin Microbiol 53:1537-1548

8. Janetanakit T, Lumyai M, Bunpapong N, Boonyapisitsopa S, Chaiyawong $\mathrm{S}$, Nonthabenjawan $\mathrm{N}$, Kesdaengsakonwut $\mathrm{S}$, Amonsin A (2016) Porcine deltacoronavirus, Thailand, 2015. Emerg Infect Dis 22:757-759

9. Jung K, Kim J, Ha Y, Choi C, Chae C (2006) The effects of transplacental porcine circovirus type 2 infection on porcine epidemic diarrhoea virus-induced enteritis in preweaning piglets. Vet J 171:445-450

10. Jung K, Scheuer KA, Zhang Z, Wang Q, Saif LJ (2014) Pathogenesis of GIII.2 bovine norovirus, CV186-OH/00/US strain in gnotobiotic calves. Vet Microbiol 168:202-207

11. Jung K, Wang Q, Scheuer KA, Lu Z, Zhang Y, Saif LJ (2014) Pathology of US porcine epidemic diarrhea virus strain PC21A in gnotobiotic pigs. Emerg Infect Dis 20:662-665

12. Jung K, Hu H, Eyerly B, Lu Z, Chepngeno J, Saif LJ (2015) Pathogenicity of 2 porcine deltacoronavirus strains in gnotobiotic pigs. Emerg Infect Dis 21:650-654

13. Jung K, Hu H, Saif LJ (2016) Porcine deltacoronavirus infection: etiology, cell culture for virus isolation and propagation, molecular epidemiology and pathogenesis. Virus Res. doi:10.1016/j. virusres.2016.04.009

14. Kim L, Chang KO, Sestak K, Parwani A, Saif LJ (2000) Development of a reverse transcription-nested polymerase chain reaction assay for differential diagnosis of transmissible gastroenteritis virus and porcine respiratory coronavirus from feces and nasal swabs of infected pigs. J Vet Diagn Invest 12:385-388

15. Lee S, Lee C (2014) Complete Genome Characterization of Korean Porcine Deltacoronavirus Strain KOR/KNU14-04/2014. Genome announcements 2
16. Li G, Chen Q, Harmon KM, Yoon KJ, Schwartz KJ, Hoogland MJ, Gauger PC, Main RG, Zhang J (2014) Full-length genome sequence of porcine deltacoronavirus strain USA/IA/2014/8734. Genome Announc 2

17. Ma Y, Zhang Y, Liang X, Lou F, Oglesbee M, Krakowka S, Li J (2015) Origin, evolution, and virulence of porcine deltacoronaviruses in the United States. mBio 6:e00064

18. Madson DM, Magstadt DR, Arruda PH, Hoang H, Sun D, Bower LP, Bhandari M, Burrough ER, Gauger PC, Pillatzki AE, Stevenson GW, Wilberts BL, Brodie J, Harmon KM, Wang C, Main RG, Zhang J, Yoon KJ (2014) Pathogenesis of porcine epidemic diarrhea virus isolate (US/Iowa/18984/2013) in 3-weekold weaned pigs. Veterinary microbiology 174:60-68

19. Marthaler D, Jiang Y, Collins J, Rossow K (2014) Complete genome sequence of strain SDCV/USA/Illinois121/2014, a porcine deltacoronavirus from the United States. Genome Announc 2

20. Marthaler D, Raymond L, Jiang Y, Collins J, Rossow K, Rovira A (2014) Rapid detection, complete genome sequencing, and phylogenetic analysis of porcine deltacoronavirus. Emerg Infect Dis 20:1347-1350

21. Oka T, Saif LJ, Marthaler D, Esseili MA, Meulia T, Lin CM, Vlasova AN, Jung K, Zhang Y, Wang Q (2014) Cell culture isolation and sequence analysis of genetically diverse US porcine epidemic diarrhea virus strains including a novel strain with a large deletion in the spike gene. Vet Microbiol 173:258-269

22. Sinha A, Gauger P, Zhang J, Yoon KJ, Harmon K (2015) PCRbased retrospective evaluation of diagnostic samples for emergence of porcine deltacoronavirus in US swine. Vet Microbiol 179:296-298

23. Song D, Zhou X, Peng Q, Chen Y, Zhang F, Huang T, Zhang T, Li A, Huang D, Wu Q, He H, Tang Y (2015) Newly emerged porcine deltacoronavirus associated with diarrhoea in swine in China: identification, prevalence and full-length genome sequence analysis. Transbound Emerg Dis 62:575-580

24. Thachil A, Gerber PF, Xiao CT, Huang YW, Opriessnig T (2015) Development and application of an ELISA for the detection of porcine deltacoronavirus IgG antibodies. PloS one 10:e0124363

25. Wang L, Byrum B, Zhang Y (2014) Detection and genetic characterization of deltacoronavirus in pigs, Ohio, USA, 2014. Emerg Infect Dis 20:1227-1230

26. Wang QH, Costantini V, Saif LJ (2007) Porcine enteric caliciviruses: genetic and antigenic relatedness to human caliciviruses, diagnosis and epidemiology. Vaccine 25:5453-5466

27. Woo PC, Huang Y, Lau SK, Yuen KY (2010) Coronavirus genomics and bioinformatics analysis. Viruses 2:1804-1820

28. Woo PC, Lau SK, Lam CS, Lau CC, Tsang AK, Lau JH, Bai R, Teng JL, Tsang CC, Wang M, Zheng BJ, Chan KH, Yuen KY (2012) Discovery of seven novel Mammalian and avian coronaviruses in the genus deltacoronavirus supports bat coronaviruses as the gene source of alphacoronavirus and betacoronavirus and avian coronaviruses as the gene source of gammacoronavirus and deltacoronavirus. J Virol 86:3995-4008 